\title{
Mode selection in a dendritelike nonlinear system
}

\author{
J. S. Langer \\ Institute for Theoretical Physics, University of California, Santa Barbara, California 93106
}

\section{H. Müller-Krumbhaar}

Institut für Festkörperforschung, Kernforschungsanlage Jülich, D-5170 Jülich, Federal Republic of Germany

(Received 21 July 1982)

\begin{abstract}
An oversimplified mathematical model that contains some of the basic features of the mode-selection problem in dendritic solidification is developed and investigated both analytically and numerically. We find that there exists a unique side-branching state of this system in which the dendrite tip is performing small oscillations about its point of marginal stability, and we argue that this state is the natural operating mode of our model. We also argue that external noise will always tend to drive the system into this state, but that purely thermal fluctuations are much too small to provide the mode-selection mechanism that is seen experimentally.
\end{abstract}

\section{INTRODUCTION}

The phenomenon of dendritic solidification presents the theorist with a number of unusual difficulties. ${ }^{1}$ The basic problem is one of mode selection. As in the related cases of Rayleigh-Bénard convection in fluids or directional solidification of alloys, the dendritic system admits a continuous family of linearly stable, steady-state forms of growth-the so-called "needle crystals" with varying tip radii and growth speeds. Real dendrites reproducibly select just one of these growth modes. The theoretical problem is to identify the mechanism by which that mode is selected and thus to predict the properties of the resulting solidification patterns. No clear solution to this problem seems known as yet for any of the situations mentioned above. In the case of the dendrite, we have argued that a combination of nonlinear effects and external noise might cause the system to operate at or near a point of marginal stability ${ }^{2}$; and this conjecture seems to be consistent with experiment. ${ }^{3-5} \mathrm{~A}$ similar conjecture regarding the directionally solidifying systems, ${ }^{6,7}$ although apparently in rough agreement with meager experimental data, seems harder to justify theoretically. ${ }^{8}$

The difficulties of the dendrite problem are compounded by geometry. In the Rayleigh-Bénard or directional solidification problems, the patterns are generally translationally symmetric_-parallel arrays of rolls or solidification cells-and this symmetry helps to simplify the analysis. No such symmetry is available for a dendrite. The geometry of a freely growing dendritic structure is relatively complex, to say the least; and descriptions of this system using parabolic coordinates lack useful simplifying features that appear in the other more familiar kinds of problems.

Associated with the low symmetry of the dendritic geometry are two features that we believe are crucial to an understanding of pattern selection in this system. First, the needle-crystal core of the dendrite can pass continuously from one steadystate configuration to another; that is, it can change its tip radius and growth speed slowly and smoothly without deviating from steady-state conditions. In contrast, cellular structures can change their spacing only by undergoing finite perturbations away from steady state, for example, the breaking of a convective roll, the splitting or pinching-off of a solidification cell, etc. It is this smoothness of the dendritic picture that may allow the marginalstability mechanism to be more effective for dendrites than for cellular structures. The other important, distinguishing, geometrical feature of the dendrite is its open-endedness. The dendrite tip, upon which we focus most of our attention, is the moving end point of a semi-infinite structure. As viewed in a frame of reference moving with the steady-state velocity of the tip, perturbations at the tip generate side-branching deformations which move back along the dendrite and grow as they move. These propagating deformations couple in an intrinsically time-dependent manner to the motion of the tip, apparently causing the system to lock into the periodic state which is the dynamically stable operating 
mode of the dendrite.

These, then, are the two main theses of this paper: that the smoothness of the dendritic geometry permits fluctuations to drive the system into the neighborhood of a point of marginal stability, and that the actual operating mode is a unique limit cycle centered near the marginal-stability point. At present, however, we are unable to develop these theses within the context of even a modestly realistic model of a dendrite. The complexity of the real system seems to preclude anything but numerical analysis, which has turned out to be extremely difficult even for linearized versions of the problem. ${ }^{9}$ Moreover, the underlying mathematical structure of the system is, so far as we know, unlike anything that has been studied previously. Our strategy, therefore, has been to study an oversimplified mathematical mode-selection problem that contains some of the basic features described above. Hopefully, this modelistic investigation will provide the understanding that we need in order to carry out more realistic calculations.

The scheme of this paper is as follows. In Sec. II, we introduce a nonlinear, fourth-order, partial differential equation that seems to us to contain most of the essential mathematical features of dendritelike mode-selection problem. Section III is devoted to a linear-stability analysis of this equation. The full nonlinear equation is still too complicated to solve analytically, but numerical analysis is relatively simple compared to what is needed for a realistic dendrite theory. Our numerical results are described in Sec. IV. Here we show that this nonlinear system, in the absence of external noise, either relaxes to one of its stable stationary states or settles into a unique oscillatory pattern very near its marginal-stability point. Finally, in Sec. V, we present a very speculative and qualitative discussion of the effects of noise on this system. Our tentative conclusion is that the predicted drift of stable stationary states toward the marginal-stability point does indeed occur, but that, to be physically significant, the intensity of the noise spectrum at wavelengths comparable to the tip radius of the dendrite must be many orders of magnitude larger than what one would expect from thermal fluctuations alone.

\section{RATIONALE FOR THE MODEL}

We start by considering a realistic linearized equation of motion for deformations of a dendritic solidification surface. For simplicity, we write this equation for the case of a two-dimensional dendrite, with vanishing thermal conductivity in the solid, and in the limit $p \ll 1$, where $p$ is the thermal Péclet number to be defined below. The equation is ${ }^{2}$

$$
(1+\xi) \frac{\partial F}{\partial \tau}=-\frac{1}{2} F-\xi \frac{\partial F}{\partial \xi}+\int_{0}^{\infty} d \xi^{\prime} G\left(\xi, \xi^{\prime}\right)\left(1+\sigma \mathscr{K}^{(1)}\right) F\left(\xi^{\prime}\right)
$$

We call (2.1) a "realistic" equation because it is expressed in terms of geometrically appropriate parabolic coordinates and its derivation is based on the diffusion kinetics which govern the motion of a solidification front. Specifically, $\xi$ is the parabolic coordinate with measures distance along the dendrite away from the tip,

$$
\xi=(r-z) / \rho
$$

where growth is in the $+z$ direction, $\rho$ is the tip radius, and $r$ is the radial distance from the origin of the coordinate system. Note that this coordinate system moves with the steady-state velocity of the tip; the steady-state needle crystal is stationary in this frame of reference. The function $F(\xi, \tau)$ measures the displacement of the solidification surface from its steady-state position, and is defined in terms of $\eta$, the parabolic coordinate orthogonal to $\xi$,

$$
\eta=(r+z) / \rho=1+F(\xi, \tau) .
$$

The scaled time $\tau$ is related to real time $t$ by

$$
\tau=2 v t / \rho,
$$

where $v$ is the growth velocity.

The integral operator $G$ which appears on the right-hand side of (2.1) is an inverse diffusion kernel evaluated in the limit that the tip radius $\rho$ is very much smaller than the diffusion length $l \equiv 2 D / v$, where $D$ is the thermal diffusion constant. The ratio of the latter two lengths if the Péclet number $p$ referred to above,

$$
p \equiv \rho / l \ll 1, l=2 D / v .
$$

For present purposes, $G$ is most usefully displayed 
as a Fourier cosine transform

$$
\begin{gathered}
G\left(\xi, \xi^{\prime}\right)=\lim _{\delta \rightarrow 0} \int_{0}^{\infty} \cos (k \sqrt{\xi}) \cos \left(k \sqrt{\xi^{\prime}}\right) \\
\times e^{-\delta k} \frac{k d k}{2 \pi \sqrt{\xi^{\prime}}} .
\end{gathered}
$$

$\mathscr{K}^{(1)}$ is the linear curvature operator in parabolic coordinates,

$$
\begin{aligned}
\mathscr{K}^{(1)}= & \frac{2 \xi}{(1+\xi)^{1 / 2}} \frac{\partial^{2}}{\partial \xi^{2}}+\frac{1+2 \xi}{(1+\xi)^{3 / 2}} \frac{\partial}{\partial \xi} \\
& +\frac{1-2 \xi}{(1+\xi)^{5 / 2}} .
\end{aligned}
$$

The last two formulas illustrate the difficulties of dealing directly with the realistic equation of motion.

The only physical parameter which has not been scaled out of (2.1) is the dimensionless group $\sigma$,

$$
\sigma=l d_{0} / \rho^{2} .
$$

Here, $d_{0}$ is the capillary length $T_{M} \gamma c / L^{2}$, where $T_{M}$ is the melting temperature, $\gamma$ the surface tension, $c$ the specific heat per unit volume, and $L$ the latent heat per unit volume. Direct numerical analysis $^{9}$ of (2.1) indicates that unstable eigenmodes appear when $\sigma$ becomes less than $\sigma^{*} \cong 0.06$. To account for heat flow in the solid (with the same diffusion constant as in the liquid), one must reduce the estimate of $\sigma^{*}$ by a factor of about 2, which is roughly consistent with the experimental estimate of $\sigma^{*} \cong 0.02$ for succinonitrile.

The marginal-stability criterion $\sigma=\sigma^{*}$ provides us via (2.8) with one relation between the growth parameters $\rho$ and $v$. In order to complete the prediction of $\rho$ and $v$ separately and-for present purposes-to guess a form for nonlinear relations, one must use this criterion in conjunction with a steady-state condition. For the two-dimensional system being considered here, the latter condition is

$$
\Delta=2 e^{p} p^{1 / 2} \int_{p^{1 / 2}}^{\infty} e^{-u^{2}} d u,
$$

where $p$ is the Péclet number defined in (2.5) and $\Delta$ is the dimensionless undercooling of the fluid far from the dendrite

$$
\Delta=\frac{T_{M}-T_{\infty}}{L / c}
$$

Note that, for fixed undercooling, (2.9) gives us simply a relation of the form $p \propto \rho v=$ const. Throughout this discussion we omit capillary corrections to the steady-state analysis, which we have shown elsewhere ${ }^{9}$ to be qualitatively unimportant.

A fully nonlinear equation of motion for the dendritic solidification surface should somehow incorporate both the family of steady states ( $\rho v=$ const) described by (2.9) and the behavior of deformations described to linear order by (2.1). The essential nonlinear effect is that a deformation $F$ implies a change in the tip radius $\rho$ and, via (2.9), a corresponding change in growth velocity $v$. These changes, in turn, imply changes in both the scaling of (2.1) and the value of $\sigma$ which appears there. These interrelations must somehow be mimicked in our nonlinear model.

It will be easiest to construct the nonlinear mode using unscaled variables, say $t, z$, and $R$, where $t$ is time, $z$ measures linear distance along the "dendrite," and $R(z, t)$ is something like the position of the solidification surface. For more detailed comparisons, we might go back to (2.2) and (2.3) and say that

$$
z \approx-\rho \xi / 2, \quad \xi \gg 1
$$

and

$$
R(z, t) \cong \rho(1+F)
$$

where $\rho$ denotes the tip radius about which one has linearized in deriving (2.1). In the nonmoving laboratory frame of reference, the "dendrite" exists in the region $z \leq z_{0}(t)$, where $z_{0}(t)$ is the position of the tip which is moving in the $+z$ direction with velocity $v(t)$. We generally shall work in the moving frame defined by the transformation

$$
z \rightarrow z-\int^{t} v\left(t^{\prime}\right) d t^{\prime}
$$

so that

$$
\frac{\partial}{\partial t} \rightarrow \frac{\partial}{\partial t}-v(t) \frac{\partial}{\partial z}
$$

In Ref. 9 we remarked that our numerical linearstability analysis seemed to predict amplification rates very roughly of the form $\left(\sigma^{*}-\sigma\right) k^{2}-k^{4}$, where $k$ is the wave number of a side-branching deformation. In fact, the circular approximation described there and the actual fit to data at large $k$ indicated that a more appropriate form might be $|k|\left(\sigma^{*}-\sigma-k^{2}\right)$. The appearance of $|k|$ instead of $k^{2}$ can be traced back to the unpleasantly singular nature of the integral representation for $G$ in (2.6)—note the factor $k$ in the cosine transform. Obviously, this singular integral kernel contains the diffusion kinetics of the realistic solidification problem, and equally obviously, we want to avoid that 
mathematical complexity in this investigation. In what follows, we shall replace the integral kernel with the simple second derivative $\partial^{2} / \partial z^{2}$, suggested by the factor $k^{2}$ in the above discussion.

Another mathematical complexity that we want to avoid is the appearance of explicitly $z$-dependent functions in our equation of motion. Explicit dependence on $\xi$ occurs throughout the realistic equations because the tip of the dendrite is geometrically distinct from other regions. For the most part, we shall simply ignore this $\xi$ dependence. As stated, we shall replace $G$ by $\partial^{2} / \partial z^{2}$, and we shall do the same for $\mathscr{K}^{(1)}$. The tip does have to be treated as a special point, however, and this will be done by imposing a boundary condition at $z=0$ (in the moving frame). The appropriate condition is suggested by thinking of $R$ as the analog of the parabolic coordinate $\eta$ in $(2.3)$ so that $R(z=0, t)$ is the instantaneous tip radius at time $t$. In order to allow needle crystals of the form $R=\rho=$ const to correspond to time-independent solutions of our proposed equation of motion, we need the condition

$$
\left.\frac{d R}{d z}\right|_{z=0}=0 \text {. }
$$

We can then incorporate the steady-state relations (2.9) and (2.10) by writing

$$
\frac{v(t) R(0, t)}{2 D}=p(\Delta),
$$

where $p(\Delta)$, a function only of dimensionless undercooling $\Delta$, can be obtained by inverting (2.9).

The above considerations leads us to propose a dendritelike nonlinear differential equation of the form

$$
\frac{\partial R}{\partial t}=v \frac{\partial R}{\partial z}-a R \frac{\partial^{2} R}{\partial z^{2}}\left[1+\epsilon \frac{\partial^{2} R}{\partial z^{2}}\right]-b \frac{\partial^{4} R}{\partial z^{4}},
$$

valid for $z<0$ and supplemented by boundary conditions (2.15) and (2.16) and others to be specified later. Note that the right-hand side of (2.17) vanishes for any constant $R$, so that we do indeed recover a continuous family of stationary solutions with tip radii and velocities related by the steadystate relations.

We believe that we have made the simplest sensible choice of the nonlinearities in (2.17). The second term on the right-hand side, $R \partial^{2} R / \partial z^{2}$, produces the basic side-branching instability and causes this instability to be stronger at larger $R$. This term produces the contribution to the linear amplification rate proportional to $\left(\sigma^{*}-\sigma\right) k^{2}$, whose sign is positive for $\sigma<\sigma^{*}$ or, equivalently, for large enough $\rho$. We have chosen this form of the nonlinearity rather than, for example, $\partial^{2} R^{2} / \partial z^{2}$ because the latter would have implied local conservation of the area under the curve $R(z)$, and there seems to be no reason for even an approximate conservation law of that kind. On the contrary, it is important that our model be sufficiently asymmetric to allow outward growth of "side branches" without equivalent melt-back in the grooves between them. We shall need this asymmetry later when we discuss the effect of fluctuations. The third-order term in $R$, proportional to $\epsilon$, is included in (2.17) to prevent the side branches from growing out to infinity in finite times. The parameter $\epsilon$ is presumably some capillary length of order $d_{0}$ or, perhaps, $d_{0} / \Delta$, the radius of the critical nucleus at undercooling $\Delta$; but our results are mostly insensitive to its precise value. The principal capillary effect in (2.17) is the fourth derivative which stabilizes small-amplitude deformations at short wavelengths. This linear term may be thought of as arising from the combined action of the integral kernel $G$ and the curvature operator $\mathscr{K}^{(1)}$, both of which are replaced here by second derivatives with respect to $z$.

The quantities $a$ and $b$ in (2.17) must be understood to be functions of the velocity $v(t)$. Their functional dependence and approximate order of magnitude may be guessed by comparing the linearized version of (2.17) with the realistic equation of motion (2.1). Using (2.4) for $t$, (2.11) for $z$, and (2.12) for $R$, we find the following linearization of (2.17):

$$
\frac{\partial F}{\partial \tau} \cong-\frac{\partial F}{\partial \xi}-\frac{2 a}{v} \frac{\partial^{2}}{\partial \xi^{2}}\left(1+\frac{4 b}{a \rho^{3}} \frac{\partial^{2}}{\partial \xi^{2}}\right) F
$$

Comparison with (2.1) suggests that

$$
\frac{2 a}{v} \cong \alpha
$$

and

$$
\frac{4 b}{a \rho^{3}} \cong \beta \sigma,
$$

where $\alpha$ and $\beta$ are numerical constants. The value of $\beta$ may be taken to be of order unity if we are trying to make a rough but direct comparison with (2.1). If we want to use (2.17) for the interpretation of succinonitrile experiments, we might try to account for heat flow in the solid by choosing $\beta \sim 2$. 
An appropriate order of magnitude for $\alpha$ can be chosen by requiring that the marginal-stability point for (2.18) occurs when $\beta \sigma=\beta \sigma^{*} \cong 0.06$. This is best accomplished by performing another scaling transformation

$$
\xi=(\alpha \beta \sigma)^{1 / 3} x, \quad \tau=(\alpha \beta \sigma)^{1 / 3} \bar{\tau},
$$

so that (2.18) becomes

$$
\frac{\partial F}{\partial \bar{\tau}}=-\frac{\partial F}{\partial x}-2 h \frac{\partial^{2} F}{\partial x^{2}}-\frac{\partial^{4} F}{\partial x^{4}}
$$

with

$$
h=\frac{1}{2}\left(\frac{\alpha^{2}}{\beta \sigma}\right)^{1 / 3} .
$$

In the following section, we show that the marginally stable value of $h$ in $(2.22)$ is $h^{*} \cong 0.36$. Then (2.23), evaluated at $\sigma=\sigma^{*}$, requires that we choose $\alpha \cong 0.14$.

The marginal-stability condition provides a convenient point of reference for scaling the nonlinear equation (2.17). The scaling relations (2.4), (2.11), and (2.12) do not make sense in the nonlinear context because $\rho$ and $v$ become variables which are directly related to $R$; specifically, we identify

$$
\rho(t)=R(0, t)
$$

and use (2.16) $(v \rho / 2 D=p)$ to evaluate $v(t)$. However, $\rho^{*}$ and $v^{*}$ are completely determined as functions of $\Delta$ by (2.16) and $\sigma=\sigma^{*}$; thus we can write

$$
t=\frac{\rho^{*}\left(\alpha \beta \sigma^{*}\right)^{1 / 3}}{2 v^{*}} \tau, \quad z=-\frac{1}{2} \rho^{*}\left(\alpha \beta \sigma^{*}\right)^{1 / 3} x
$$

and

$$
\begin{aligned}
R(z, t) & =\rho^{*}\left(\frac{\beta \sigma^{*}}{\alpha^{2}}\right)^{1 / 3} \widetilde{R}(x, \tau) \\
& =\left(\frac{\rho^{*}}{2 h^{*}}\right) \widetilde{R}(x, \tau) .
\end{aligned}
$$

The dimensionless factors involving $\alpha, \beta$, and $\sigma^{*}$ have been introduced in order to be consistent with the notation used in (2.22). Equation (2.17) becomes

$$
\frac{\partial \widetilde{R}}{\partial \tau}=-v \frac{\partial \widetilde{R}}{\partial x}-v \widetilde{R} \frac{\partial^{2} \widetilde{R}}{\partial x^{2}}\left(1+\widetilde{\epsilon} \frac{\partial^{2} \widetilde{R}}{\partial x^{2}}\right)-\frac{1}{v} \frac{\partial^{4} \widetilde{R}}{\partial x^{4}},
$$

where

$$
v(\tau)=\frac{v}{v^{*}}=\frac{2 h^{*}}{\widetilde{R}(0, \tau)}
$$

and

$$
\tilde{\epsilon}=\frac{4 \epsilon}{\rho^{*} \alpha\left(\alpha \beta \sigma^{*}\right)^{1 / 3}} .
$$

The boundary conditions for (2.27) can be chosen to be

$$
\begin{aligned}
\widetilde{R}^{\prime}(0, \tau) & =\widetilde{R}^{\prime \prime \prime}(0, \tau) \\
& =\widetilde{R}^{\prime}(L, \tau)=\widetilde{R}^{\prime \prime \prime}(L, \tau)=0,
\end{aligned}
$$

where primes denote differentiation with respect to $x$, and the boundary at $x=L$ is to be moved to infinity: $L \rightarrow \infty$. The first-derivative conditions are required, as mentioned above, in order that constant values of $\widetilde{R}$ be acceptable solutions. The third derivatives are chosen arbitrarily. Note that if we write

$$
\widetilde{R}=2 h(1+F)
$$

and linearize (2.27) in $F$, we recover (2.22). Note also that the only free parameter in (2.27) is $\widetilde{\epsilon}$. If $\epsilon$ is of order $d_{0} / \Delta$, then $\widetilde{\epsilon}$ will be of order unity.

\section{LINEAR-STABILITY THEORY}

In this section we present an account of the linear-stability analysis which produces the value of $h^{*}$ quoted following (2.23). The discussion is not necessary for an understanding of the material in later sections of this paper. However, these results do provide some useful insight into the behavior of the realistic linear equation (2.1); thus, we shall describe the analysis in some detail.

We start with the linear equation (2.22). If we write

$$
F(x, \bar{\tau})=f_{W}(x) e^{W \bar{\tau}},
$$

then the relevant eigenvalue problem is

$$
W f_{W}=-\frac{\partial f_{W}}{\partial x}-2 h \frac{\partial^{2} f_{W}}{\partial x^{2}}-\frac{\partial^{4} f_{W}}{\partial x^{4}}
$$

with

$$
f_{W}^{\prime}(0)=f_{W}^{\prime \prime \prime}(0)=f_{W}^{\prime}(L)=f_{W}^{\prime \prime \prime}(L)=0, L \rightarrow \infty .
$$

This is not a standard kind of eigenvalue equation; the linear operator on the right-hand side of (3.2) contains a fourth derivative and, because of the first 
derivative, is not self-adjoint.

The general solution of (3.2) must have the form

$$
f_{W}(x)=\sum_{n=1}^{4} A_{n} \exp \left[i K_{n}(W) x\right]
$$

where the $K_{n}(W)$ are the four solutions of

$$
M(K, W) \equiv K^{4}-2 h K^{2}+i K+W=0 .
$$

From the boundary conditions (3.3) we obtain the determinantal eigenvalue equation

$$
\mathscr{D}(W)=\operatorname{det}\left(\begin{array}{cccc}
i K_{1} & i K_{2} & i K_{3} & i K_{4} \\
-i K_{1}^{3} & -i K_{2}^{3} & -i K_{3}^{3} & -i K_{4}^{3} \\
i K_{1} e^{i K_{1} L} & i K_{2} e^{i K_{2} L} & i K_{3} e^{i K_{3} L} & i K_{4} e^{i K_{4} L} \\
-i K_{1}^{3} e^{i K_{1} L} & -i K_{2}^{3} e^{i K_{2} L} & -i K_{3}^{3} e^{i K_{3} L} & -i K_{4}^{3} e^{i K_{4} L}
\end{array}\right)=0
$$

Suppose that $\operatorname{Im} K_{1} \leq \operatorname{Im} K_{2} \leq \operatorname{Im} K_{3} \leq \operatorname{Im} K_{4}$. That is, let $\exp \left(i K_{1} x\right)$ be the most rapidly growing exponential function, $\exp \left(i K_{2} x\right)$ the next most rapid, etc. Then group the terms in (3.6) as follows:

$$
\begin{aligned}
\mathscr{D}(W)= & Q_{1}(W) e^{i\left(K_{1}+K_{2}\right) L}+Q_{2}(W) e^{i\left(K_{1}+K_{3}\right) L} \\
& +Q_{3}(W) e^{i\left(K_{2}+K_{3}\right) L}+\cdots
\end{aligned}
$$

where

$$
Q_{1}(W)=K_{1} K_{2} K_{3} K_{4}\left(K_{2}^{2}-K_{1}^{2}\right)\left(K_{4}^{2-} K_{3}^{2}\right),
$$

etc. The first two terms on the right-hand side of (3.7) are the largest in the limit of indefinitely large $L$. If $\operatorname{Im} K_{2}<\operatorname{Im} K_{3}$, then the only solutions in this limit are fixed by setting $Q_{1}=0$; but this relation gives us only isolated points (e.g., $W=0$, $f_{0}=$ constant) and not a full spectrum of eigenvalues. The way to obtain the spectrum in the large- $L$ limit is to set

$$
\operatorname{Im} K_{2}=\operatorname{Im} K_{3}
$$

so that the first two terms in (3.7) remain of comparable size no matter how large $L$ becomes.

The relation (3.9) is sufficient to determine a full (presumably complete) spectrum of complex eigenvalues $W$ and eigenfunctions $f_{W}(x)$. Note that this relation does not depend on the precise nature of the boundary conditions (3.3), but only on the fact that we are imposing two conditions at $x=0$ and two at $x=L \rightarrow \infty$. Only the special modes determined by $Q_{1}=0$ are strongly boundary dependent. The eigenfunctions themselves must look as follows. Let the amplitude $A_{2}$ in (3.4) be unity. Then $A_{1}$, the coefficient of the most rapidly growing term in (3.4), must be small, of order $\exp \left[-\left(\operatorname{Im} K_{2}-\operatorname{Im} K_{1}\right) L\right]$, so that the first three terms in (3.4) all are of compar- able size near $x=L$. Similarly, the second, third, and fourth terms in (3.4) all will have magnitudes of order unity near $x=0$. The absence of a cubic term in (3.5) implies that

$$
\sum_{n=1}^{4} K_{n}=0
$$

which tells us that at least one of the terms in (3.4), specifically the fourth, must be a decaying exponential which may be appreciable near $x=0$ but drops out near $x=L$. In this way, we are able to adjust $W$ and the three independent amplitudes $A$ so as to fit the four boundary conditions, two at end.

The eigenvalue spectra obtained by imposing the condition (3.9) on the solutions of (3.5) are illustrated in Fig. 1 for $h=1, h=h^{*} \cong 0.3622$, and $h=0$. Here, both the real and imaginary parts of $W$ are shown as functions of the real part of $K, K$ being either $K_{2}$ or $K_{3}$ as explained below. Each spectrum consists of two parts: a complex branch shown by a solid line starting at $\operatorname{Re} K=0$, and a real branch shown by a separate line starting at larger values of $\operatorname{Re} K$. For both of these branches, $K$ is chosen to be $K_{2}$; and we have adopted the convention that $\mathrm{ReK}_{2} \leq \operatorname{Re} K_{3}$. The dashed segments in Fig. 1(a) are second (redundant) representations of the complex branches, shown here with $K=K_{3}$. When that latter segments are included, the spectra appear as piecewise continuous functions of $K$ and look qualitatively like those obtained for the realistic model in Ref. 9. The results of $\operatorname{Im} W$ shown in Fig. 1(b) are also similar to these in Ref. 9, with wave velocities $\operatorname{Im} W / \operatorname{Re} K$, which are very nearly unity near the peak in the spectrum and are somewhat larger at smaller $K$. Note that the marginally stable branch of the spectrum, with $h=h^{*}$; passes through its maximum with $\operatorname{Re} W=0$ at a finite value of $\operatorname{Re} K$. 

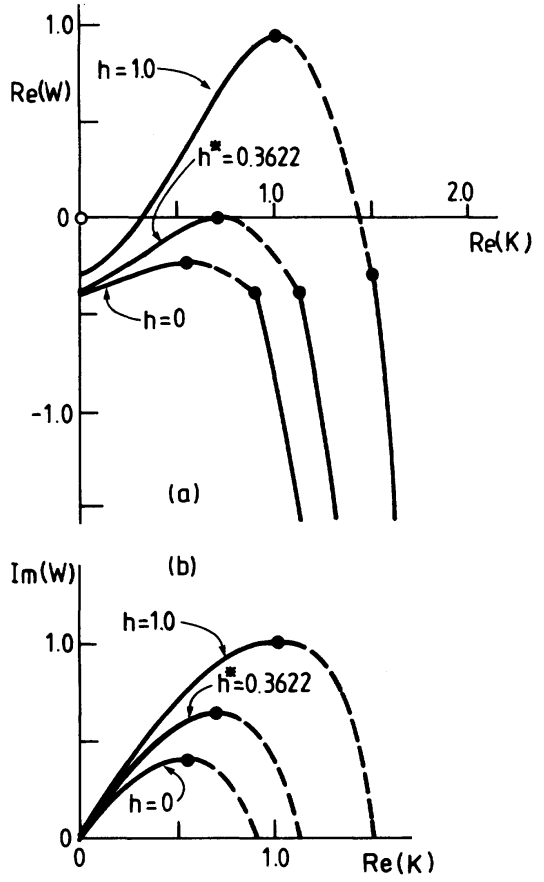

FIG. 1. Eigenvalue spectra for the linear-stability problem at three different values of the parameter $h$.

The existence of such a characteristic length at the marginal-stability point was unclear in the earlier numerical results.

In the complex part of the spectrum, the modes $f_{W}$ are superpositions of two dominant, moving, growing waves with distinct wave numbers $\mathrm{Re} K_{2}$ and $\mathrm{ReK}_{3}$, both positive. The transition to the real part of the spectrum occurs when $\operatorname{Re} K_{2}$ vanishes and $K_{2}$ and $K_{1}$ exchange roles. The real modes $f_{W}$ are standing waves with only a single wave number $\operatorname{Re} K_{2}=-\operatorname{Re} K_{3}, \operatorname{Re} K_{1}=\operatorname{Re} K_{4}=0$, which grow in $x$ and decay exponentinally in time. The most interesting points for our purposes are the peaks, indicated by open circles in Fig. 1(a). These peaksmaxima in $\mathrm{Re} W$-occur when $K_{2}$ merges with $K_{3}$, that is, when (3.5) has a double root. The latter condition can be used to compute $h^{*}$ and to deduce properties of the spectra near the peaks.

To see how this calculation is performed, note first that when $K_{2}=K_{3}$, the polynomial $M(K)$ defined in (3.5) can be written

$$
M(K, \bar{W})=\left(K-\bar{K}_{1}\right)\left(K-\bar{K}_{2}\right)^{2}\left(K-\bar{K}_{4}\right),
$$

where the quantities with overbars $\bar{W}, \bar{K}_{i}$ indicate that the equation is valid only when $K_{2}=K_{3}$. Equating coefficients of the various powers of $K$ in
(3.5) and (3.11), we can express $\bar{K}_{1}$ and $\bar{K}_{4}$ in terms of $\bar{K}_{2}$ and then obtain the relations

$$
\begin{aligned}
& 2 \bar{K}_{2}^{3}-2 h \bar{K}_{2}+\frac{i}{2}=0, \\
& \bar{W}=3 \bar{K}_{2}^{4}-2 h \bar{K}_{2}^{2},
\end{aligned}
$$

which can be solved for $\bar{W}$ and $\bar{K}_{2}$ as functions of $h$. The marginal-stability point is identified by the value of $h=h^{*}$ such that $\operatorname{Re} \bar{W}=0$. After some algebra, we find that this point occurs when $K_{2}=K^{*}$,

$$
\begin{aligned}
K^{*} & =\left(\frac{3+\sqrt{7}}{32}\right)^{1 / 6} \exp \left[-\frac{i}{2} \cos ^{-1}\left(\frac{\sqrt{7}-1}{2}\right)\right] \\
& \cong 0.7150-0.2229 i
\end{aligned}
$$

and that

$$
h^{*}=\left(\frac{1+\sqrt{7}}{2}\right)\left(\frac{3-\sqrt{7}}{4}\right)^{2 / 3} \cong 0.3622 .
$$

To check that the condition $K_{2}=K_{3}$ does in fact identify a maximum in $\operatorname{Re} W$, we can write

$$
\begin{aligned}
M(K, W) \cong & \left(K-\bar{K}_{2}\right)^{2}\left(\bar{K}_{2}-\bar{K}_{1}\right)\left(\bar{K}_{2}-\bar{K}_{4}\right) \\
& +W-\bar{W}=0
\end{aligned}
$$

for $W$ near $\bar{W}$ and $K$ near $\bar{K}_{2}$. The roots of (3.16) are

$$
K \cong K_{2} \pm\left(\frac{\bar{W}-W}{P(h)}\right)^{1 / 2},
$$

where

$$
P(h) \equiv\left(\bar{K}_{2}-\bar{K}_{1}\right)\left(\bar{K}_{2}-\bar{K}_{4}\right)=2\left(3 \bar{K}_{2}^{2}-h\right) .
$$

These two roots are now to be identified as the distinct values of $K_{3}$ and $K_{2}$, respectively. The condition that they have the same imaginary part can be satisfied, in this approximation, only if the quantity inside the large parentheses in (3.17) is real and positive. Note then that $\operatorname{Im} K_{2}=\operatorname{Im} K_{3}=\operatorname{Im} K^{*}$. Thus, the spectrum is approximated by

$$
W \cong \bar{W}-P(h)\left(\operatorname{Re} K-\operatorname{Re} \bar{K}_{2}\right)^{2},
$$

where $K$ is $K_{2}$ or $K_{3}$ depending upon whether $\operatorname{Re} K$ is less than or greater than $\operatorname{Re} \bar{K}_{2}$. The condition for a maximum is simply that $\operatorname{Re} P(h)$ be greater 
than zero, which is true in the region of interest, as seen in (3.21) below.

\section{NONLINEAR BEHAVIOR}

We return now to the fully nonlinear problem defined by Eqs. (2.27) and (2.28) and the boundary conditions (2.30). Our investigation has consisted primarily of a sequence of numerical experiments supplemented by a simple two-mode analytic interpretation of the numerical results.

The integration of Eq. (2.27) was performed by discretization in the $x$ direction with 200 (and sometimes 400 ) points, the distance between meshpoints mostly being $\Delta x=0.7$. This discretization allowed us to study systems containing about 14 sidebranching periods at a resolution of about fourteen meshpoints per period. We used a standard fivepoint Runge-Kutta procedure [IMSL (Information Management Systems Library) subroutine DVERK] with double precision on an IBM 3033 computer. Computation times were as long as several hours per run in some cases to allow for extrapolation to the long-time limit.

Boundary conditions were chosen as follows. At $x=0$, all odd derivatives were set equal to zero as specified in (2.30). At $x=L=200 \Delta x$, however, we set $\widetilde{R}(x>L)=\widetilde{R}(L)$ and, in the interval $L-10 \Delta x<x<L$, introduced a linearly progressive changeover from the original version of (2.27) to a pure diffusion equation with a drift term. The purpose of this procedure was to minimize reflection of signals at the large- $x$ boundary and thus simulate a semi-infinite system. An alternative procedure would have been to construct a reflection-free boundary condition for the dominant modes of the Fourier spectrum of $\widetilde{R}$, but this would have required an additional time-consuming computation which did not seem to be necessary in view of the apparent success of our simpler method. We did check our method by performing some computations on a larger system with 400 meshpoints.

Our typical procedure was to integrate $(2.27)$ forward in time starting with $\widetilde{R}$ of the form

$$
\widetilde{R}(x, 0)=\widetilde{R}_{0}+r_{0}(x) .
$$

Here, $\widetilde{R}_{0}$ was chosen to have various values on either side of the marginally stable $\widetilde{R}_{0}^{*}=2 h^{*} \cong 0.72$, and $r_{0}(x)$ was some initial perturbation localized in the neighborhood of the tip, that is near $x=0$. In all of these numerical experiments, we observed just two qualitatively different kinds of behavior: either the system settled eventually to a uniform stationary state with $\widetilde{R}$ equal to some constant less than
0.72 , all perturbations having moved out of the system in the direction of large $X$, or else the system settled into a unique, time-dependent, sidebranching state whose properties were independent of the details of the initial configuration. The first kind of behavior generally occurred when the initial $\widetilde{R}_{0}$ was less than 0.72 and $r_{0}(x)$ was not too large; but we occasionally observed the system to find its way to such a uniform state after undergoing large excursions in the apparently unstable regime. Similarly, the second kind of behavior generally, but not always, occurred when the initial $\widetilde{R}_{0}$ was larger than 0.72 .

A sequence of pictures showing the evolution of the side-branching state from an initially unstable configuration is shown in Fig. 2. The initial perturbation, shown in Fig. 2(a), grows and moves in the $+x$ direction, as seen in $2(\mathrm{~b})$, and also generates new oscillations near $x=0$. In the final state, shown in 2(d), the side branches are continuously generated by a small-amplitude oscillation at the tip, and grow to some $\widetilde{\epsilon}$-dependent steady-state amplitude as they move down the dendrite.

Within a side-branching wavelength of the tip, the amplitude of oscillation of the steady state, shown in Fig. 2(c), is extremely small. In fact, the amplitude of oscillation of the tip displacement $\widetilde{R}(x=0, \tau)$ in our numerical experiments was only of order $10^{-2}$ for values of $\widetilde{\epsilon}$ of order unity or greater. This is consistent with experimental pictures $^{4}$ which show visible side-branching deformations emerging well behind the smooth, paraboloidal tips of succinonitrile dendrites. This feature has also allowed us to simplify our study of the steady-state behavior. Because the steady state $\widetilde{R}(0, \tau)$ is very nearly a constant, the factors $\nu(\tau)$ which appear on the right-hand side of (2.27) are also nearly constant and play essentially no role after the initial transients have settled out. Thus, in all of the numerical work to be discussed next, we have simply set $v=1$ in (2.27) and have ignored (2.28).

One of the main purposes of this investigation was to test the validity of the marginal-stability hypothesis which implies, in part, that the steady-state tip displacement $\widetilde{R}(0, \tau)$ should oscillate around or near the value $2 h^{*}=0.72$. This test requires a careful study of the long-time behavior of the system in order to be sure that the steady state has actually been reached. The approach to steady state is also of some interest by itself.

In order to suppress irrelevant degrees of freedom, we analyzed the time dependence of $\widetilde{R}(0, \tau)$ by producing a Poincaré map. Each time $\widetilde{R}(0, \tau)$ went 

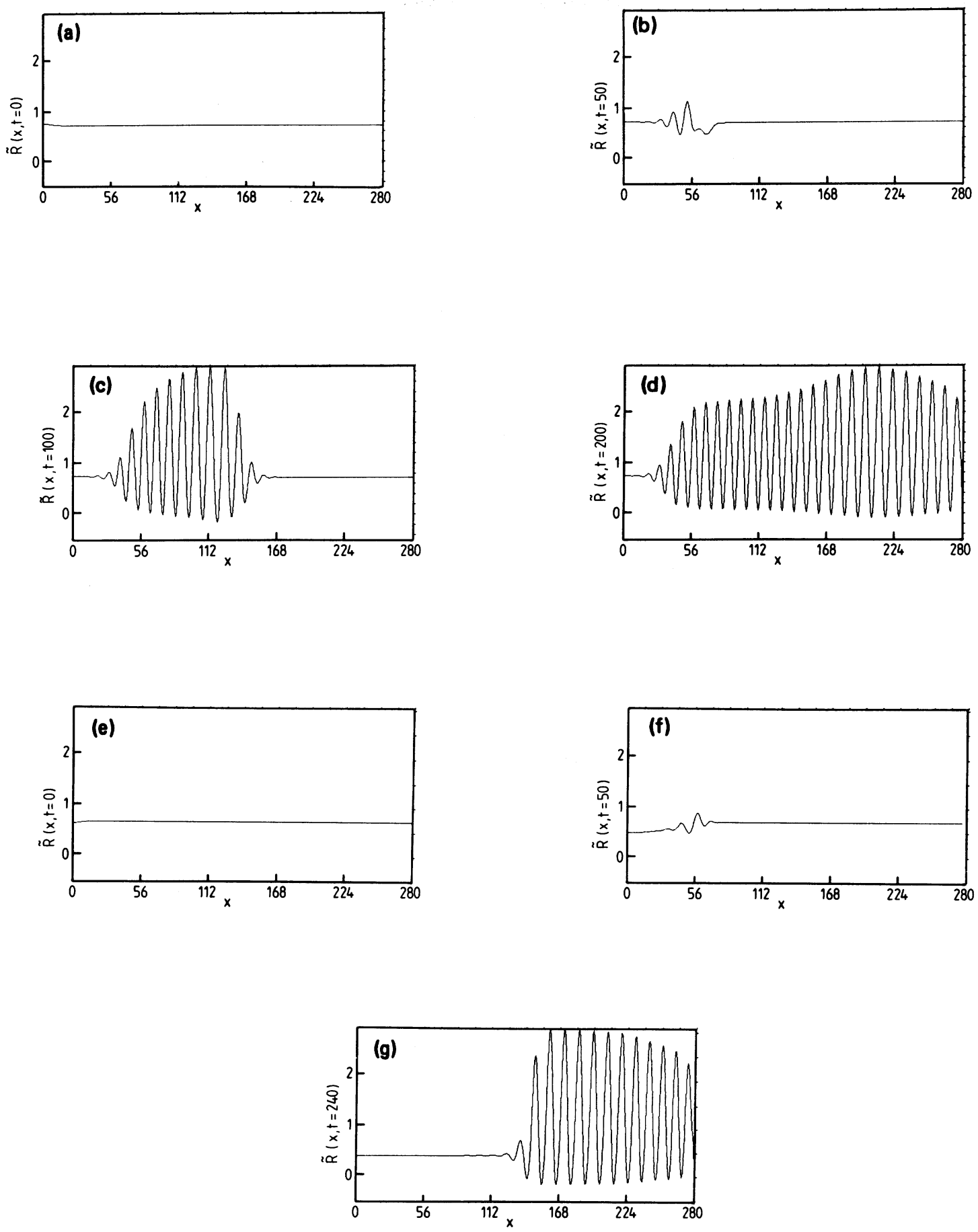

FIG. 2. (a) -(d) Sequence of pictures showing the evolution of the side-branch pattern from the marginally stable configuration with an initial destabilizing perturbation near the tip. (e)-(g) same as (a) - (d), but with an initial stabilizing perturbation near the tip. Even though side branches are formed, their wave front cannot keep up with the tip velocity and the dendrite ultimately returns to a smooth shape, in the absence of fluctuations. 
through a maximum, we determined its value $\widetilde{R}_{\max }\left(0, \tau_{n}\right)$ and the corresponding $\tau_{n}$ by parabolic interpolation between three successive time steps. After steady state was reached, this procedure yielded constant values $\widetilde{R}_{\max }\left(0, \tau_{n}\right)$ and the period $\tau_{n}-\tau_{n-1}$ to an accuracy of five significant figures.

In Fig. 3 we show the results of this analysis for $\tilde{\boldsymbol{\epsilon}}=0.5$. Each dot represents a maximum of the tip amplitude, and the intermediate motion is not shown. The initial value is $\widetilde{R}_{0}=0.85$ [see Eq. (4.1)]. The asymptotic value is $\widetilde{R}_{\max }(0, \infty) \cong 0.897$, which is reached with exponential covergence. The oscillations seen in the figure have a period of roughly $\Delta \tau \cong 130$. This is of the same order of magnitude as the time it takes for a signal to travel the length of the system, but this long-period oscillatory behavior does not seem to be entirely a size effect. Doubling the size of the system from 200 to 400 meshpoints with constant $\Delta x$ did increase the period, but only to $\Delta \tau \cong 150$. At this relatively small value of $\widetilde{\epsilon}$, the oscillations near the tip are large, and the side branches away from the tip have such large amplitudes that $\widetilde{R}(x, \tau)$ becomes negative in the troughs. We conclude that this is an unphysically small value of $\widetilde{\epsilon}$, and we have not investigated such small $\widetilde{\epsilon}$ 's any further.

When $\tilde{\epsilon}$ is greater than 0.7 , the behavior of $\tilde{\boldsymbol{R}}_{\max }\left(0, \tau_{n}\right)$ becomes quite different, as is seen in Fig. 4. Here, for $\widetilde{\epsilon}=1.0$, the long-period oscillation has disappeared and the tip displacement relaxes smoothly toward a value below 0.75 . The long-time convergence is no longer exponential, however. To show this more clearly, we have made the doublelogarithmic plot shown in Fig. 5. In preparing this graph, we have adjusted the value of $\widetilde{R}_{\max }(0, \infty)$ so as to obtain a straight line corresponding to a power law of the form

$$
\widetilde{R}_{\max }\left(0, \tau_{n}\right)-\widetilde{R}_{\max }(0, \infty) \propto \tau_{n}^{-\mu} .
$$

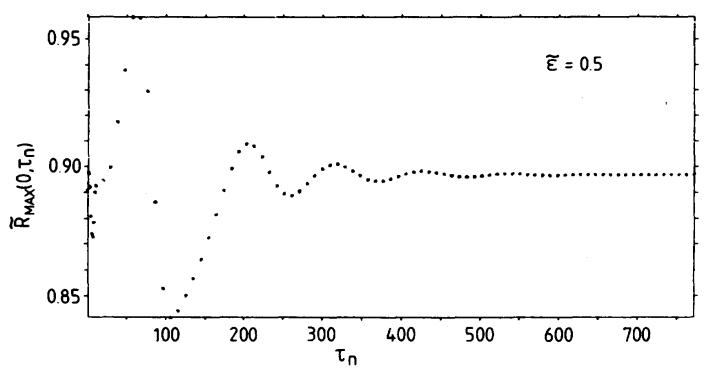

FIG. 3. Graph of maximum tip displacements $\widetilde{R}_{\max }\left(0, \tau_{n}\right)$, showing oscillatory relaxation toward steady state at $\widetilde{\boldsymbol{\epsilon}}=0.5$.

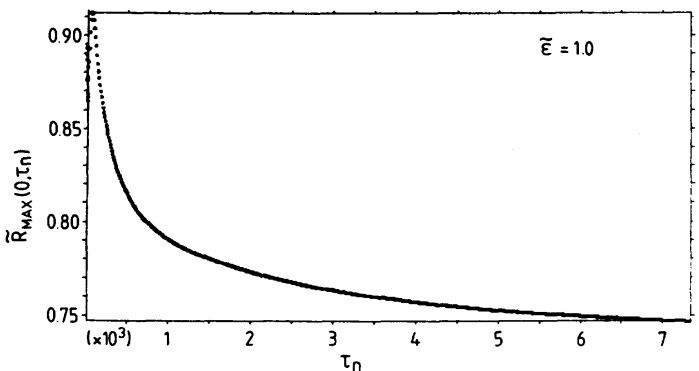

FIG. 4. Graph of maximum tip displacements $\widetilde{R}_{\max }\left(0, \tau_{n}\right)$, showing slow, nonoscillatory relaxation toward steady state at $\widetilde{\boldsymbol{\epsilon}}=1.0$.

We find $\widetilde{R}_{\max }(0, \infty) \cong 0.72 \pm 0.01$ and $\mu=0.61$. Other values of $\widetilde{\epsilon}$ between 0.7 and 5.0 gave the same qualitative behavior. In particular, $\widetilde{R}_{\max }\left(0, \tau_{n}\right)$ always relaxed to 0.72 as above, except for $\widetilde{\epsilon}=0.7$, where it was about 0.75 . The amplitude of the tip oscillation, $\tilde{R}_{\max }(0, \tau)-R_{\max }(0, \tau)$, was approximately 0.02 for $\widetilde{\epsilon}=1.0$ and decreased for increasing $\widetilde{\epsilon}$. The exponent $\mu$ decreased with increasing $\tilde{\epsilon}$, becoming approximately 0.38 at $\widetilde{\epsilon}=2.0$ and 0.33 at $\widetilde{\boldsymbol{\epsilon}}=3.0$. Accordingly, the time needed for $\widetilde{R}_{\max }\left(0, \tau_{n}\right)$ to come within $5 \%$ of the asymptotic value increased to about $2 \times 10^{4}$ at $\tilde{\epsilon}=3.0$. During this time, some 2000 side branches had grown out of the tip region.

The side-branching patterns at the ends of four runs with different $\tilde{\epsilon}$ 's are shown in Fig. 6. All tip displacements $\widetilde{R}_{\max }(0, \tau)$ have converged to values near 0.75 . Beyond a few wavelengths from the tip, both the average displacements $\widetilde{R}(x, t)$ and the amplitudes of the side-branching oscillations have settled to values which decrease with increasing $\widetilde{\epsilon}$. Note that the spacings of the side branches, like the tip displacements, are essentially independent of $\tilde{\boldsymbol{\epsilon}}$. (The relative phases of the side-branching oscillations in Fig. 6 are not significant. These pictures have been drawn in the moving frame of reference at arbitrarily different phases of their side-

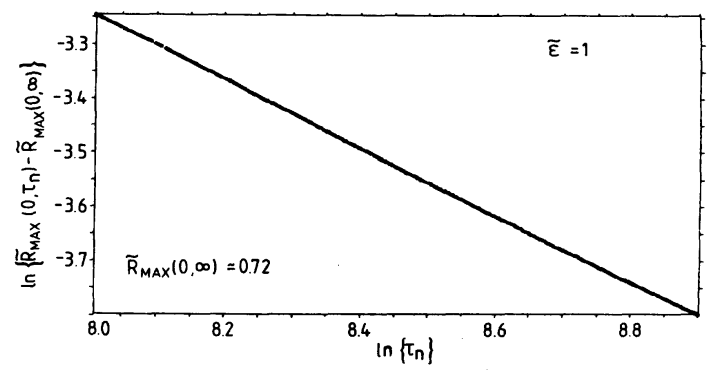

FIG. 5. Logarithmic plot of the relaxation of the maximum tip displacements at $\widetilde{\boldsymbol{\epsilon}}=\mathbf{1 . 0}$. 


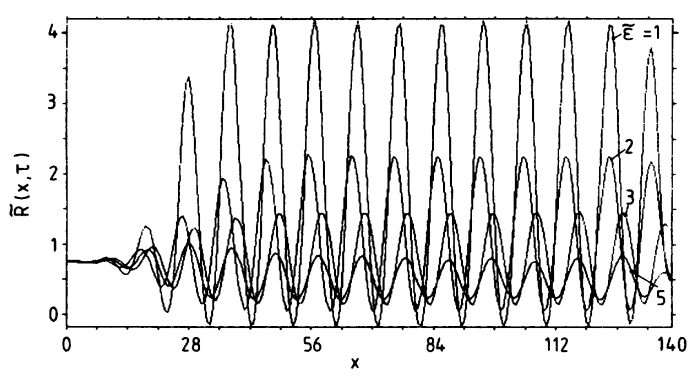

FIG. 6. Side-branching patterns at the ends of four runs with different values of $\widetilde{\epsilon}$ as shown.

branching limit cycles.)

Figure 6 illustrates several of the central results of this investigation. The tip displacement $\widetilde{R}(0, \tau)$ clearly settles into a small-amplitude oscillation within a $1 \%$ neighborhood of the point of marginal stability, as can be deduced from the initially slow spatial growth of side branches away from the tip. This limit cycle is unique in the sense that it appears to be produced by any starting configuration other than those which lead back to the stable regime, $\widetilde{R}=$ const $<0.72$. Moreover, this behavior seems to be essentially independent of $\widetilde{\epsilon}$, the one remaining system parameter in (2.27). The spacing of the side branches seems to be completely determined by the oscillation at the tip and, thus, like the tip displacement, to be independent of initial conditions and $\widetilde{\epsilon}$. We believe that these features are also characteristic of the real physical situation, and that the emergence of these features in our model provides new understanding of the marginal-stability hypothesis.

In the remainder of this section, we shall describe a two-mode analytic interpretation of our numerical results which is motivated largely by the above observations regarding the side-branching modes. The side-branching patterns shown in Fig. 6 suggest that, for values of $x$ far from the tip, the Fourier decomposition

$$
\widetilde{R}(x, \tau) \cong r_{0}+\sum_{m=1}^{\infty} r_{m} \cos \left[m k_{0}(x-v \tau)\right]
$$

should be a rapidly convergent series. The value of $k_{0}$ deduced from Fig. 6 is about 0.65 , just slightly less then $\operatorname{Re} K^{*}=0.715$ in (3.14). Our strategy is to consider states nearly but not quite at equilibrium by allowing the $r$ 's in (4.3) to be slowly $\tau$ dependent, and by truncating this expansion at $m=1$. We then look for coupled, nonlinear equations for $r_{0}(\tau)$ and $r_{1}(\tau)$.

Observing the side branches far from the tip, in the laboratory frame of reference, we might consid- er simply ignoring the boundary conditions. That is, we could eliminate the drift term at $x=0$ by writing $y=x-v t$ and

$$
\widetilde{R}(y, \tau) \cong r_{0}(\tau)+r_{1}(\tau) \cos k_{0} y .
$$

If we then substituted (4.4) into (2.27) and interpreted the results as if it were valid on the infinite interval, $-\infty<y<\infty$, we could project out truncated equations of motion for $r_{0}$ and $r_{1}$. Doing this, however, would lose the information about the stability of the system for $r_{0}<2 h^{*} \cong 0.72$ and would also introduce an inconsistency in our assumptions about $k_{0}$. To avoid these difficulties we propose adding a term linear in $r_{1}$ which will mimic the known stability properties. Our equation, valid in some region $x=y+v t \gg 1$, is

$$
\begin{aligned}
\frac{\partial \widetilde{R}}{\partial \tau}= & \left.-c r_{1} \cos k_{0} y-\widetilde{R} \frac{\partial^{2} \widetilde{R}}{\partial y^{2}} \mid 1+\widetilde{\epsilon} \frac{\partial^{2} \widetilde{R}}{\partial y^{2}}\right) \\
& -\frac{\partial^{4} \widetilde{R}}{\partial y^{4}},
\end{aligned}
$$

where the first term on the right-hand side is the new stabilizing factor and the rest comes directly from (2.27). If we choose $c=h^{* 2}$, then marginal stability occurs at $r_{0}=2 h^{*}$ as it should. The critical $k_{0}$ is $\sqrt{h^{*}} \cong 0.6$, which is just slightly smaller than either the observed value $(0.65)$ or $\operatorname{Re} K^{*}=0.75$. Because (4.5) is only a one-parameter approximation to the full equation, we cannot expect to reproduce both $r_{0}$ and $k_{0}$ exactly.

The resulting equations of motion for the $r$ 's are

$$
\begin{aligned}
& \frac{d r_{0}}{d t}=\frac{1}{2} h^{*} r_{1}^{2}\left(1-\widetilde{\epsilon} h^{*} r_{0}\right), \\
& \frac{d r_{1}}{d t}=h^{*} r_{1}\left(r_{0}-2 h^{*}\right)-\frac{3}{4} \widetilde{\epsilon} h^{* 2} r_{1}^{3} .
\end{aligned}
$$

This model has a line of fixed points at $r_{1}=0$ and, for $\widetilde{\epsilon}<\widetilde{\epsilon}_{c}=\frac{1}{2} h^{* 2} \cong 3.8$, another fixed point at

$$
r_{0}^{*}=\frac{1}{\tilde{\epsilon} h^{*}}, \quad r_{1}^{*}=\frac{1}{\tilde{\epsilon} h^{*}}\left[\frac{4}{3}\left(1-2 \widetilde{\epsilon} h^{* 2}\right)\right]^{1 / 2} .
$$

The fixed line is stable with respect to perturbations in the $r_{1}$ direction for $r_{0}<2 h^{*}$ and unstable otherwise. As shown in Fig. 7, the isolated fixed point (4.7) merges with the fixed line at $r_{0}=2 h *$ when $\widetilde{\epsilon}$ increases to $\widetilde{\epsilon}_{c}$.

For $\widetilde{\epsilon}<\widetilde{\epsilon}_{c}$, our analysis leads us to conclude that the ultimately stable state of the system is at the isolated side-branching fixed point (4.7). If the system starts with small, nonzero $r_{1}$ and $r_{0}<2 h^{*}$, then 

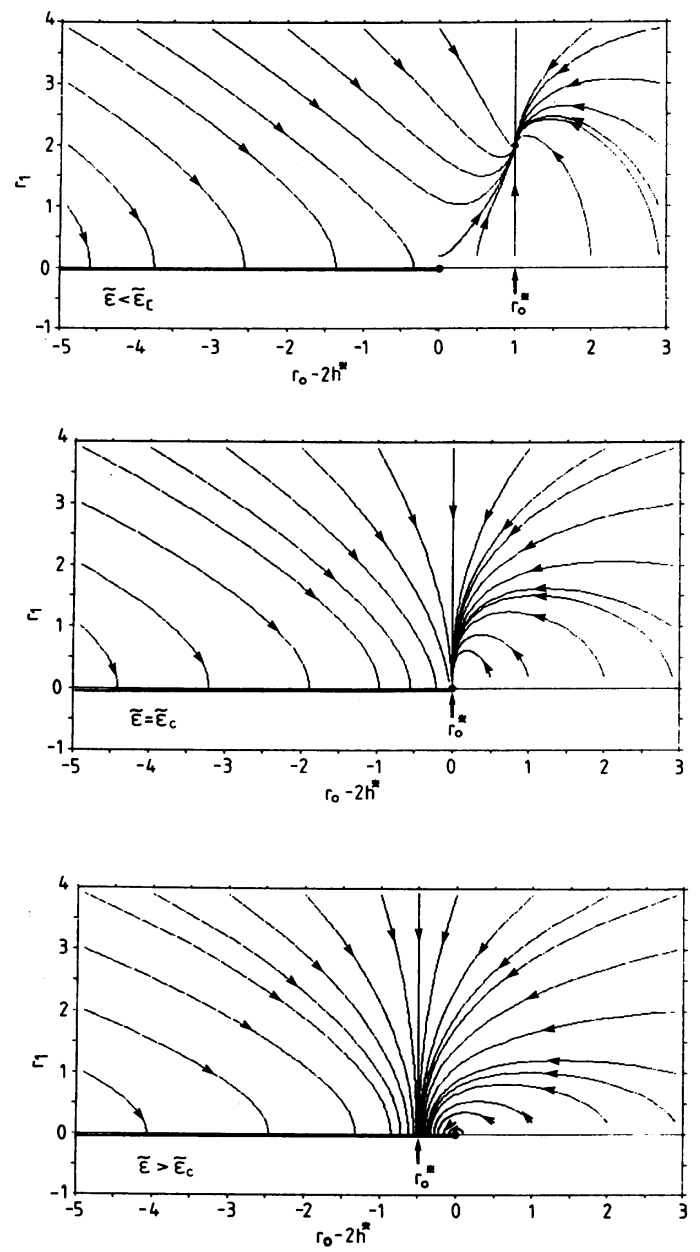

FIG. 7. Flow diagrams for Eqs. (4.6) for three different values of $\widetilde{\boldsymbol{\epsilon}}$.

$r_{1}$ decays exponentially and the system settles at some point on the fixed line. Such a point is stable against side-branching deformations but is only marginally stable against changes in $r_{0}$, that is, against changes in the average curvature of the dendrite. If $r_{1}$ is prevented from decaying to zero by some additional noise source, $d r_{0} / d t$ remains positive and the system drifts toward $r_{0}=2 h^{*}$. This drift was anticipated in our earlier papers. ${ }^{2}$ The effect is also found in numerical studies of (2.27) with added noise (not reported here), and is implicit in the fluctuation theory to be described in Sec. V. Finally, if the system finds itself near the unstable part of the fixed line $r_{0}>2 h^{*}$, then it moves directly to the side-branching fixed point (4.7).

When $\widetilde{\boldsymbol{\epsilon}}=\widetilde{\boldsymbol{\epsilon}}_{c}$ [Fig. $7(\mathrm{~b})$ ], the isolated fixed point has just merged with the fixed line at $r_{0}=2 h^{*}$. If the system starts in the unstable range $r_{0}>2 h^{*}$, the side-branching amplitude $r_{1}$ decays like $\tau^{-1 / 2}$ rather than exponentially, but the side branches ultimately disappear. When $\widetilde{\epsilon}>\widetilde{\epsilon}_{c}$, all points near the unstable part of the fixed line are mapped onto the interval $1 / \tilde{\boldsymbol{E}} h^{*}<r_{0}<2 h^{*}$. Even though a smooth initial condition with $r_{0}>2 h^{*}, r_{1}=0$ is unstable against side-branching deformations, it ultimately stabilizes to a state where the side branches are suppressed.

We conclude by comparing the predictions of this rudimentary model (4.6) with our results from (2.27). First, we have observed numerically that the side-branching mode predicted by (2.27) equilibrates with a power law dependence on time; and this is in contrast to the simpler model (4.6) which predicts exponential relaxation everywhere except exactly at $\widetilde{\boldsymbol{\epsilon}}_{c}$. Apparently the simplified model (4.6) misses some subtle details of the tip dynamics. Second, the simplified model predicts a critical $\widetilde{\epsilon}_{c}=3.8$ above which the side branches should decay. This is not seen in the calculations based on (2.27). The graph for $\widetilde{\epsilon}=5.0$ in Fig. 6 shows that the average displacement $r_{0}$ at large $x$ does fall below the marginal stability value, even though $\widetilde{R}(0, \tau)$ seems to remain at 0.72 . The equilibrium time at such a large $\widetilde{\epsilon}$ is so long, however, that the system may not have reached steady state. For most values of $\widetilde{\epsilon}$ between 1 and 5, the agreement between our simple analysis and the numerical results is quite good, as is shown in Fig. 8. In particular, the decay of the homogeneous mode $r_{0}$ below the critical value $2 h^{*}$ at $\widetilde{\epsilon}=\widetilde{\epsilon}_{c}$ is well reproduced. We have therefore obtained a fairly good representation for the mechanism of sidebranch stabilization in our nonlinear model (2.27) for the physical range of values of $\widetilde{\epsilon}$ of order unity.

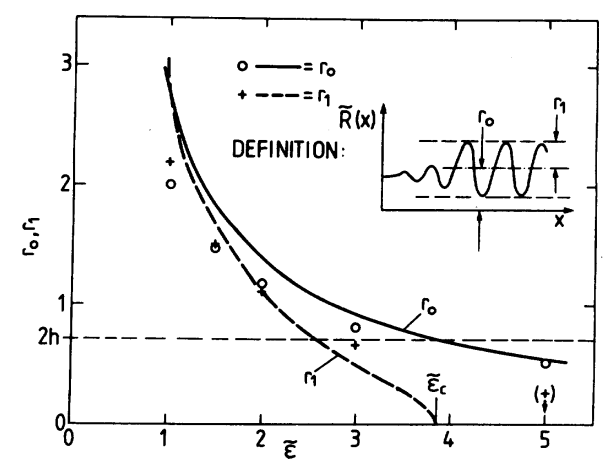

FIG. 8. Steady-state amplitudes $r_{0}, r_{1}$ as functions of $\widetilde{\boldsymbol{\epsilon}}$. Circles are numerical data, lines are results from the simplified model (4.6). 


\section{v. FLUCTUATIONS} fluctuation effects, especially in the continuum of stable states $\left(\sigma>\sigma^{*}\right)$ where we expect fluctuations to produce a drift toward the point of marginal stability $\left(\sigma=\sigma^{*}\right)$. The following discussion will be even more qualitative and speculative than the preceding sections.

Our general strategy is to add a Langevin force $\eta(z, t)$ to the right-hand side of the nonlinear equation of motion (2.17) and to use this equation to estimate a fluctuation-driven rate of change of the tip radius $\rho=R(0)$, in the stable regime $\rho<\rho^{*}$. This program consists of two main parts. First, we must choose an appropriate stochastic description of the random force $\eta$, and then we have to make some estimate for the effect of these fluctuations on $\rho$. A direct solution of the complete Langevin equation, (2.17) plus the noise term $\eta$, seems neither feasible nor necessary at the present stage of the investigation; thus we shall make some very crude approximations designed only to look for general trends and orders of magnitude.

Let us start with the assumption that the underlying source of fluctuations is thermal noise. We shall see that it is unlikely that purely thermal fluctuations can produce the effect that we are looking for; but they provide a convenient starting point, and it is far less clear how to describe any other possible noise sources. So far as we know, the only previous theory of the effects of thermal fluctuations on solidification is that of Cherepanova, ${ }^{10}$ who has calculated the spectrum of thermally driven deformations of the face of a growing crystal near the point where it undergoes a Mullins-Sekerka instability. ${ }^{11}$ Cherepanova's published results are not directly useful for us because she provides only a formula for the interfacial fluctuations of an initially planar solidification front rather than an expression for the stochastic force $\eta$ which we might borrow for use in other situations. We shall, however, follow her general procedure, but in a much simpli-
We turn, finally, to the problem of estimating

fied form.

Specifically, we consider the somewhat artificial situation of a pure solid growing into a melt with unit undercooling; $\Delta=\left(T_{M}-T_{\infty}\right) c / L=1$. That is, the undercooling is just large enough to allow steady-state planar growth at any velocity. We further specialize to the case in which the thermal diffusion constants $D$ and specific heats $c$ are the same in both liquid and solid phases. Then we can write the equation of motion for the thermal diffusion field, in the frame of reference moving with the interface in the $z$ direction at velocity $v$, in the form

where

$$
\frac{\partial u}{\partial t}=D \nabla^{2} u+v \frac{\partial u}{\partial z}+S
$$

$$
u=\frac{T-T_{M}}{L / c}
$$

is the dimensionless temperature and $S$ is a stochastic heat source whose correlation coefficients are more conveniently written in terms of its Fourier components $\hat{\boldsymbol{S}}(\overrightarrow{\mathrm{k}}, t)$ :

$$
\begin{aligned}
&\left\langle\hat{S}(\overrightarrow{\mathrm{k}}, t) \hat{S}\left(-\overrightarrow{\mathrm{k}}^{\prime}, t^{\prime}\right)\right\rangle \\
&=\frac{2 k_{B} T^{2} c D k^{2}}{L^{2}} \delta\left(\overrightarrow{\mathrm{k}}-\overrightarrow{\mathrm{k}}^{\prime}\right) \delta\left(t-t^{\prime}\right) .
\end{aligned}
$$

Note that (5.1) is valid throughout the two-phase system and that (5.3) reproduces the correct thermal fluctuations in either phase because of the uniformity of $D$ and $c$. Note also that $\vec{k}$ is a threedimensional wave vector. ( $k_{B}$ is Boltzmann's constant.)

Let $\vec{x}$ be a vector in the plane of the interface (perpendicular to the $z$ axis), let $\zeta(\vec{x}, t)$ be the displacement of the interface in the $z$ direction, and consider a displacement of the form

$$
\zeta(\overrightarrow{\mathrm{x}}, t)=\hat{\zeta}\left(\overrightarrow{\mathrm{k}}_{1}\right) \exp \left(i \overrightarrow{\mathrm{k}}_{1} \cdot \overrightarrow{\mathrm{x}}+i \omega t\right),
$$

where $\overrightarrow{\mathbf{k}}_{1}$ is a two-dimensional wave vector in the $\overrightarrow{\mathbf{x}}$ plane. The appropriate form of the solution of (5.1) is

$$
u(\vec{x}, z, t)=\left\{\begin{array}{l}
\exp \left(-\frac{2 z}{l}\right)-1+\hat{u}(z) \exp \left(i \overrightarrow{\mathbf{k}}_{\perp} \cdot \overrightarrow{\mathbf{x}}+i \omega t\right), \quad z>\zeta \text { (liquid) } \\
\hat{u}^{\prime}(z) \exp \left(i \overrightarrow{\mathbf{k}}_{\perp} \cdot \overrightarrow{\mathbf{x}}+i \omega t\right), \quad z<\zeta \text { (solidd) }
\end{array}\right.
$$

where, as before, $l=2 D / v$ is the diffusion length and

$$
\begin{aligned}
& \hat{u}(z)=\hat{U} e^{-q z}+\hat{u}_{S}(z), \\
& \hat{u}^{\prime}(z)=\hat{U}^{\prime} e^{q^{\prime} z}+\hat{u}_{S}(z) .
\end{aligned}
$$

Here

$$
u_{S}(z)=\int_{-\infty}^{\infty} \frac{d k_{z}}{2 \pi} \frac{\hat{S}\left(\overrightarrow{\mathrm{k}}_{1}, k_{z}\right) \exp \left(i k_{z} z\right)}{i \omega+D\left(k_{\perp}^{2}+k_{z}^{2}\right)-i v k_{z}}
$$


is the fluctuation-induced part of the temperature field, and $q$ and $q^{\prime}$ are the solutions (with positive real parts) of

$$
\begin{aligned}
& q^{2}-\frac{2 q}{l}-k_{\perp}^{2}-\frac{i \omega}{D}=0, \\
& q^{\prime 2}+\frac{2 q^{\prime}}{l}-k_{\perp}^{2}-\frac{i \omega}{D}=0 .
\end{aligned}
$$

The amplitudes $\hat{U}$ and $\hat{U}^{\prime}$ are easily determined to linear order in the deformation $\hat{\xi}$ by imposing the Gibbs-Thomson condition

$$
u(z=\zeta)=-d_{0} \nabla^{2} \zeta
$$

and the Stefan condition

$$
v+\frac{\partial \zeta}{\partial t}=-D\left[\frac{\partial u}{\partial z}\right]_{\zeta},
$$

where the square brackets on the right-hand side denote the discontinuity (liquid-solid) at the interface. (See Ref. 1, Sec. III A.) The resulting linear equation for $\hat{\xi}$ is

$$
i \omega \hat{\xi}\left(\overrightarrow{\mathrm{k}}_{\perp}\right)=\Omega\left(k_{\perp}\right) \hat{\xi}\left(\overrightarrow{\mathrm{k}}_{1}\right)-D\left(q+q^{\prime}\right) \hat{u}_{S}(0),
$$

where

$$
\Omega\left(k_{1}\right)=v\left(q-\frac{2}{l}\right)-D d_{0}\left(q+q^{\prime}\right) k_{\perp}^{2}
$$

is the Mullins-Sekerka amplification rate in the absence of noise.

For present purposes, it is sufficient to use a quasistationary approximation and set $\omega=0$ wherever it appears on the right-hand side of (5.11), and also to assume that $v$ is small so that $k_{1} l \gg 1$. Then $q \cong q^{\prime} \cong\left|k_{1}\right|$, and (5.11) can be written in the form

$$
\frac{\partial \hat{\xi}\left(\overrightarrow{\mathbf{k}}_{\perp}\right)}{\partial t} \cong v\left|k_{\perp}\right|\left(1-d_{0} l k_{\perp}^{2}\right) \hat{\xi}\left(k_{\perp}\right)+\hat{\eta}_{2}\left(\overrightarrow{\mathrm{k}}_{\perp}, t\right),
$$

which is a linear Langevin equation of the kind that we are looking for. The random force is

$$
\hat{\eta}_{2}\left(\overrightarrow{\mathrm{k}}_{1}, t\right) \cong 2\left|k_{\perp}\right| \int_{-\infty}^{\infty} \frac{d k_{z}}{2 \pi} \frac{\hat{s}\left(\overrightarrow{\mathrm{k}}_{1}, k_{z}, t\right)}{k_{\perp}^{2}+k_{z}^{2}}
$$

so that, using (5.3), we find

$$
\begin{aligned}
\left\langle\hat{\eta}_{2}\left(\overrightarrow{\mathrm{k}}_{\perp}, t\right) \hat{\eta}_{2}(\right. & \left.\left.-\overrightarrow{\mathrm{k}}_{\perp}^{\prime}, t^{\prime}\right)\right\rangle \\
& \cong \frac{4 k_{B} T^{2} c D}{L^{2}}\left|k_{\perp}\right| \delta\left(\overrightarrow{\mathrm{k}}_{\perp}-\overrightarrow{\mathrm{k}}_{\perp}^{\prime}\right) \delta\left(t-t^{\prime}\right) .
\end{aligned}
$$

The subscript on $\hat{\eta}_{2}$ is included in order to emphasize that this force acts over a two-dimensional surface.

Equation (5.13) has some of the features of the realistic dendrite model. Of course, neither the parabolic geometry of the dendrite tip or the drift term which causes side branches to move relative to the tip are present here, but the factor $\left(1-d_{0} l k_{\perp}^{2}\right)$ is essentially the same as $\left(1+\sigma \mathscr{K}^{(1)}\right)$ in $(2.1)$, and the factor $\left|k_{1}\right|$ is the planar (Fourier transformed) version of the inverse diffusion kernel $G$. The transition from (2.1) to (2.17), in effect, requires the replacement of the nonanalytic factor $\left|k_{1}\right|$ by $\alpha \rho k_{\perp}^{2}$, with $k_{\perp}^{2}$ being equivalent to a second derivative and $\alpha \cong 0.14$. We propose, therefore, to make the same replacement on the right-hand side of (5.15) in order to obtain a random force suitable for use with (2.17). The last modification of $\hat{\eta}_{2}$ that must be made before it can be used in (2.17) is to make its argument one-dimensional. We do this by returning to the $\overrightarrow{\mathrm{x}}$ representation $\overrightarrow{\mathrm{x}}=\left(x_{1}, x_{2}\right)$, and integrating over $x_{2}$ across a strip of width $2 \pi \rho$, that is, we average over the circumference of the dendrite. Finally, we return to the notation $x_{1}=z$, where $z$ is the distance along the "dendrite" as used in Sec. II. Our random force is therefore

$$
\eta(z, t)=\int_{0}^{2 \pi \rho} \frac{d x_{2}}{2 \pi \rho} \eta_{2}\left(x_{1}=z, x_{2}, t\right)
$$

and

$$
\begin{aligned}
& \left\langle\eta(z, t) \eta\left(z^{\prime}, t^{\prime}\right)\right\rangle \\
& =-\alpha \Lambda \frac{\partial^{2}}{\partial z^{2}} \delta\left(z-z^{\prime}\right) \delta\left(t-t^{\prime}\right),
\end{aligned}
$$

where

$$
\Lambda \cong \frac{k_{B} T^{2} c D}{\pi^{2} L^{2}}
$$

Note that $\Lambda$ turns out to be independent of either $v$ or $\rho$; thus our noise is purely additive in the sense that $\eta$ does not depend on the state of the system.

To estimate the effect of the fluctuations on the dendrite, consider performing both a spatial and a statistical average on (2.17) in a situation in which the mean value of $R(z, t)$ is a constant, for example, 
$R=\rho<\rho^{*}$. The latter condition implies that we are in the stable regime. If we neglect the cubic nonlinearity and perform an integration by parts for the spatial average, we find

$$
\frac{d \rho}{d t} \cong \frac{\alpha v}{2}\left\langle\left(\frac{\partial R}{\partial z}\right)^{2}\right\rangle .
$$

This expression has the general features that we expected; the drift in $\rho$ is in the direction of the marginal-stability point and is caused by a fluctuation-driven nonlinearity in the system. Note, that as mentioned in the paragraph following (2.17), the choice of an area nonpreserving quadratic nonlinearity was necessary in order to obtain a nonvanishing term in (5.19).

The dimensionless quantity of physical interest is $v^{-1} d \rho / d t$, which should not be too much less than unity if the marginal-stability point is to be reached in a small number of side-branching periods. To evaluate this quantity, we shall assume that $\rho$ is far enough below $\rho^{*}$ that we can use a linearized fluctuation theory. It is convenient to return to the dimensionless notation introduced in (2.21) through (2.23) and used in the linear analysis of Sec. III. We have

$$
\frac{1}{v} \frac{d \rho}{d t}=\frac{8 h^{2}}{\alpha}\left\langle\left(\frac{\partial F}{\partial x}\right)^{2}\right\rangle,
$$

where $F$ satisfies the equation

$$
\frac{\partial F}{\partial \bar{\tau}}=-\frac{\partial F}{\partial x}-2 h \frac{\partial^{2} F}{\partial x^{2}}-\frac{\partial^{4} F}{\partial x^{4}}+\bar{\eta}(x, \bar{\tau})
$$

and

$$
\begin{aligned}
& \left\langle\bar{\eta}(x, \bar{\tau}) \bar{\eta}\left(x^{\prime}, \bar{\tau}^{\prime}\right)\right\rangle \\
& =-\bar{\Lambda} \frac{\partial^{2}}{\partial x^{2}} \delta\left(x-x^{\prime}\right) \delta\left(\bar{\tau}-\bar{\tau}^{\prime}\right)
\end{aligned}
$$

with

$$
\bar{\Lambda}=\frac{16 h^{2} \Lambda}{v \rho^{4} \alpha} .
$$

One point of immediate difficulty with the fluctuation problem posed by the above set of equations is that the quantity $\left\langle(\partial F / \partial x)^{2}\right\rangle$ appears to be ultraviolet divergent. This should not be too surprising. Even bulk quantities like $\left\langle u^{2}\right\rangle$ or $\left\langle(\nabla u)^{2}\right\rangle$ computed from (5.1) and (5.3) make sense only if one specifies a fluctuation volume, that is, a shortwavelength cutoff; the solidification problem defined by $(5.13)-(5.15)$ similarly requires a cutoff for the interpretation of interfacial quantities like $\left\langle\zeta^{2}\right\rangle$ or $\left\langle(\nabla \zeta)^{2}\right\rangle$. Intuitively, however, we do not expect a new microscopic length to enter our formula for $d \rho / d t$. On the contrary, it seems reasonable to expect that the fluctuations that drive changes in $\rho$ are on the scale of $\rho$ itself. We shall take the latter point of view in our interpretation of the formulas in the next several paragraphs.

Assuming that we can either solve or avoid the divergence problem, we predict on dimensional grounds that $\left\langle(\partial F / \partial x)^{2}\right\rangle$ must have the form $\bar{\Lambda} \phi(h)$, where $\phi(h)$ is a function of order unity. That is,

$$
\frac{1}{v} \frac{d \rho}{d t} \cong \frac{128 h^{4} \Lambda}{v \rho^{4} \alpha^{2}} \phi(h)
$$

In the neighborhood of the dendritic operating point, the prefactor of $\phi$ on the right-hand side of (5.24) has the form $\left(\rho_{0} / \rho\right)^{2}$, where

$$
\rho_{0}^{2}=\left(\frac{64 h^{* 4} \sigma^{*}}{\pi^{2} \alpha^{2}}\right)\left(\frac{k_{B} c T_{M}^{2}}{L^{2} d_{0}}\right) .
$$

For succinonitrile, $\rho_{0} \cong 2 \times 10^{-8} \mathrm{~cm}$. [See Ref. 5 for estimates of the quantities in the second set of parentheses in (5.25).] The dendrites observed by Glicksman and his collaborators ${ }^{3,4}$ have tip radii $\rho$ in the range $10^{-4}$ to $10^{-2} \mathrm{~cm}$; thus the prefactor in (5.24) is at most of order $10^{-8}$ and becomes as small as $10^{-12}$ for the slower, fatter dendrites seen in the experiments. These estimates are the basis of our conclusion that purely thermal fluctuations should play no significant role in the dendritic modeselection process.

There are, of course, other irregularities in any solidifying system which might provide fluctuations with the strength that we need at length scales of the order of $\rho$. One specially interesting possibility is that these irregularities might be inherent in a more complete dynamical model of the dendritic system, perhaps arising via hydrodynamic coupling between the moving solidification front and the surrounding fluid. With such possibilities in mind, it seems to us to be useful to explore the fluctuation theory in a little more detail, that is, to look a bit further at the function $\phi(h)$.

A formal expression for $\phi(h)$ can be derived from (5.21) and (5.22) by standard methods of fluctuation theory. We find

$$
\begin{gathered}
\phi(h)=\sum_{W, W^{\prime}} \frac{d f_{W}}{d x} \frac{d f_{W^{\prime}}}{d x} \frac{1}{|W|+\left|W^{\prime}\right|} \\
\times \int_{0}^{\infty} d x_{1} \frac{d \tilde{f}_{W}}{d x_{1}} \frac{d \tilde{f}_{W^{\prime}}}{d x_{1}},
\end{gathered}
$$


where the $x$ dependence, if any remains after the sums are performed, is to be averaged out. The functions $f_{W}(x)$ denote the right eigenfunctions defined in (3.2), and the $\widetilde{f}_{W}(x)$ are their conjugates, that is, the left eigenfunctions of the same linear operator. The eigenvalues $W, W^{\prime}$ are all negative because, by choice, we are looking only in the stable regime. Instead of attempting the ambitious task of evaluating (5.26) with the exact states computed in Sec. III, let us simply approximate these states by plane waves with wave numbers $K$, and let us further approximate the dispersion relation $W(K)$ by the relation between $\operatorname{Re} W$ and $\operatorname{Re} K$ obtained in (3.19). That is,

$$
W(K) \cong \mu^{*}\left(h-h^{*}\right)-v^{*}\left(K-K_{0}^{*}\right)^{2}+\cdots,
$$

where $v^{*}=\operatorname{Re} P\left(h^{*}\right), K_{0}^{*} \cong \operatorname{Re} K^{*} \cong 0.7150$, etc. In this way, we find

$$
\phi(h) \sim \int \frac{d K}{4 \pi} \frac{K^{4}}{|W(K)|},
$$

which is linearly divergent because $W(K)$ must be proportional to $K^{4}$ at large $K$. If we simply ignore this divergence and assume that the integral is determined by its behavior near the peak at $K=K_{0}^{*}$, we find

$$
\phi(h) \sim \frac{K_{0}^{* 4}}{4\left[\mu^{*} v^{*}\left(h^{*}-h\right)\right]^{1 / 2}} .
$$

One way of interpreting this result is to note that the fluctuation-affected regime, that is, the regime in which the right-hand side of (5.24) is of order unity, occurs only when $\left(h^{*}-h\right) \sim\left(\rho^{*}-\rho\right) / \rho^{*}<10^{-16}$.

\section{ACKNOWLEDGMENTS}

This research was supported by the U.S. Department of Energy, by a NATO traval grant, and by NSF Grant No. PHY77-27084. Computations were performed at Kernforschungsanlage Jülich. One of the authors (H.M.K.) wishes to acknowledge a summer visit (1981) at the Institute for Theoretical Physics, Santa Barbara, during which part of this work was performed.
1J. S. Langer, Rev. Mod. Phys. 52, 1 (1980).

2J. S. Langer and H. Müller-Krumbhaar, Acta Metall. $\underline{26}, 1681$ (1978); 26, 1689 (1978); 26, 1697 (1978).

${ }^{3}$ M. E. Glicksman, R. J. Shaefer, and J. D. Ayers, Metall. Trans. A7, 1747 (1976).

4S. C. Huang and M. E. Glicksman, Acta Metall. 29, 701 (1981); 29, 717 (1981).

5J. S. Langer, R. F. Sekerka, and T. Fujioka, J. Cryst. Growth 44, 414 (1978).

6J. S. Langer, Phys. Rev. Lett. 44, 1023 (1980).
7J. S. Langer, Ann. N.Y. Acad. Sci. 373, 179 (1981).

8J. S. Langer, in Nonlinear Phenomena at Phase Transitions and Instabilities, edited by T. Riste (Plenum, New York, 1982), p. 309.

${ }^{9}$ H. Müller-Krumbhaar and J. S. Langer, Acta Metall. $\underline{29}, 145$ (1981).

10T. A. Cherepanova, Dok. Akad. Nauk SSSR 226, 1066 (1976) [Sov. Phys.-Dokl. 21, 109 (1976)].

${ }^{11}$ W. W. Mullins and R. F. Sekerka, J. Appl. Phys. 35, 444 (1964). 